\title{
Numerical Investigation of Flow and Heat Transfer Characteristics in Plate with Multiple Incline Stage Holes
}

\author{
Bo ZHANG, Yuanyuan DOU, Quan HONG, Honghu JI
}

\begin{abstract}
In this paper, the effects of impingement and film composite cooling on the design of combustion chamber cooling structure are simulated by numerical simulation. The focus of the investigation was on increased film cooling efficiency and enhanced heat transfer between the coolant and the hole wall. The five-stage shaped hole model and one cylindrical hole design have the same equivalent flow area. The flow and heat transfer characteristics of cylindrical hole and stage-shaped hole were numerically investigated under same blowing ratio, and compared at the same blowing ratio. The results showed the stage-shaped hole resulted in higher cooling effectiveness, especially in rear part, and the mechanisms of which were studied in details. The consequences of the phase parameters in the flow have very clearly dependedt on the internal shape of the corresponding hole. Stage-shaped holes formed impact inside the wall, tapped the coolant potential in cooling, and increased the heat transfer inside the solid wall. Further, stage-shaped hole resulted in unstabilized flow inside hole, gave an enhancement of lateral spreading ability, and brought a significant increase of the film lateral effectiveness. Further, the affection of area ratio and height ratio has been studied by five models. The results show that the increasing of area ratio leads to an increase in cooling efficiency, which also indicates the increasing of height ratio showed slight affection.
\end{abstract}

Keywords: film cooling; heat transfer enhancement; impingement; stage-shaped holes

\section{INTRODUCTION}

With further development of aero engines, designing the combustion, chamber is facing increasing challenges from higher working temperature and heat capacity than ever. The thermal effectiveness and the specific power of the gas turbine systems depend strongly on turbine inlet temperature [1-3].

The inlet temperature limited by the potential structural failure of the engine components is mainly attributable to high temperature. This undoubtedly leads to a series of problems, such as the match between oil and air, the control of temperature distribution of combustion and the effective cooling of the combustor liner, and so on. In order to improve the reliability of the combustor liner at ever-increasing temperatures, a useful method is to devise more efficient combustor liner cooling systems, to which the effusion cooling system belongs. As alternative solutions to the typical 2-Dslot combustor cooling systems, the full coverage film cooling showed its high effective layer of film cooling characteristics [4, 5]. Andreini [6] compared the heat transfer characteristics of slot film and effusion cooling systems, and found the later ones show a significant increasing. Researchers have done many investigations for full coverage film cooling, such as: Che Sidik et al. [7], Ligrani et al. [8], Schulz [9], Kelly, and Bogard [10]; these investigations demonstrate that substantial improvement in cooling effectiveness is achieved by increasing the number of holes and simultaneously decreasing the diameter. Krewinkel [11] indicates that two key factors, which alter film cooling effectiveness, are inclination angle and spacing. Andrews et al. [12] investigated the effects of various factors on fullcoverage film cooling and showed the strong influence of the number of holes, of their length and injection angle.

Based on effusion cooling structure, impingement/ effusion cooling system was designed, which is composed of effusion and impingement cooling plates. It raises the cooling effectiveness to extent, and gets higher cooling effectiveness by approximately $30 \%$, with small coolant magnitude [13-14].
Experimental and numerical approaches have studied the cooling effectiveness of impingement/ effusion cooling. A large number of simulations, experiments, and optimization techniques have focused on enhancing the heat transfer and thermal performance of impingement jet cooling and film cooling [15-16]. The heat transfer and flow characteristics have been examined as functions of various variables, including the Reynolds number, the blowing ratio, height to diameter, number of holes, arrangement, diameter, and angle of the holes [17].

However, its complicated structure brought great difficulties to manufacture and the double wall brought extra weight, so engine designers demanded reduced weight and volume to make smaller and more effective aircraft engines. It is necessary to develop a simple and effective cooling structure.

A new scheme was developed simplified on the basis of the original imp/eff structure by merging the two plates into one, saving many connected components. It tries to increase the heat transfer between coolant and wall by forming impinging flat inside the film holes, and tap the cooling potential of the coolant gas, which is to lower the wall temperature not only through the film cooling. Nevertheless, through so-called "heat sink effect", that is the wall cooling due to the heat removed by the passage of the coolant through the holes $[8,12]$. Further, the structure of stage holes can induce unestablished flow, enhancing the lateral spreading of the film downstream.

Its flow and heat transfer characteristics have been numerically investigated and compared with the effusion cooling structure. In addition, the effect of area ratio and height ratio has been investigated.

\section{COMPUTATION SCHEME}

Fig. 1 showed the specific structures of two models. Modell was an inclined cylindrical hole with fixed flowing area, and Model 2 was composed of upstream hole and downstream hole, marked as $D_{\text {up }}$ and $D_{\text {down }}$ separately, which had variable flowing area inside the hole. Further, $D_{\text {up }}$ was smaller than $D_{\text {down, }}$ so an impact plate was formed 
inside Model 2. The two holes of Model 2 had same inclined angles $\alpha$ as $30^{\circ}$.
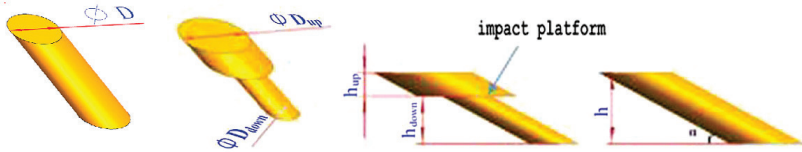

$\begin{array}{ll}\text { (a) cylindrical } & \text { (b) stages }\end{array}$

Figure 1 Sketch of effusion and stages of effusion cooling structure

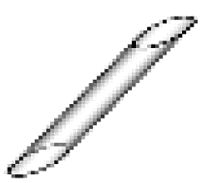

(a) Model1

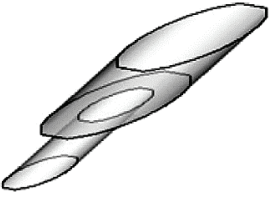

(d) Model4

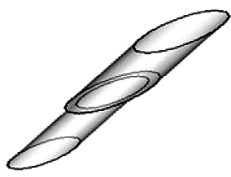

(b) Model 2

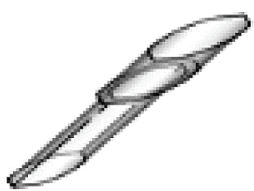

(e) Model5

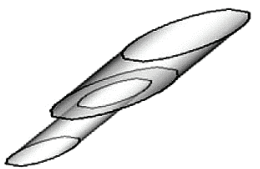

(c) Model3

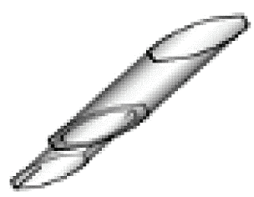

(f) Model6
Figure 2 Sketch of stage hole structures with different area and height ratio

In the paper, length ratio was defined as

$L_{\text {ratio }}=\frac{H_{\text {up }}}{H_{\text {down }}}$

and area ratio was defined as

$A_{\text {ratio }}=\frac{A_{\text {up }}}{A_{\text {down }}}$

Thus, a sudden contraction was formed at the interaction position of the hole $\mathrm{A}$ and hole $\mathrm{B}$, and an impact platform was formed in the junction of the two holes. The cooling gas first formed impingement on the flat, enhanced the convective heat transfer between coolant and the wall of the stage hole. Finally, it flew out, formed a film-cooling sheet on the wall surface.

The equivalent diameter was defined as Eq. (3).

$$
D_{0}=\frac{1}{\sqrt{\left(\frac{1}{D_{\text {up }}}\right)^{2}+\left(\frac{1}{D_{\text {down }}}\right)^{2}}}
$$

\subsection{Geometrical of the Computational Model}

The geometrical parameters of six models investigated in the paper were listed in Tab. 1, which were all designed with the same equivalent diameter as Model 1.

Models 1, 2, 3 are different in $D_{\text {up }}, D_{\text {down }}$ and same in holes length, which were designed for investigation of different area ratio influence.

Models 2, 5, 6 are different in $H_{\text {up }} / H_{\text {down }}$, and the same in hole diameter, and were designed for the investigation of different height ratio influence. The geometrical parameters of all the models were listed in Tab. 1.
Table 1 Geometrical parameters

\begin{tabular}{|c|c|c|c|c|}
\hline Model & Area ratio & $D_{\text {up }}(\mathrm{mm})$ & $D_{\text {down }}(\mathrm{mm})$ & Length ratio \\
\hline M1 & $1: 1$ & 0.6 & 0.6 & 1 \\
\hline M2 & $1.7: 1$ & 0.84 & 0.64 & 1 \\
\hline M3 & $3: 1$ & 1.09 & 0.63 & 1 \\
\hline M4 & $4: 1$ & 1.22 & 0.61 & 1 \\
\hline M5 & $1.7: 1$ & 0.84 & 0.64 & 0.5 \\
\hline M6 & $1.7: 1$ & 0.84 & 0.64 & 2 \\
\hline
\end{tabular}

\section{GRID GENERATION AND INDEPENDENCE}

The software ANSYS ICEM-CFD has been used to generate the computational grids. The domain meshes were hexahedral ones including 4 layers close to the wall, counting about 2.5 million elements in total (Seen in Fig. 3 ), which were realized with an increasing refinement level in order to perform an assessment of the mesh sensitivity. Fig. 4 presents the grid independency study, carried out with Model 2.
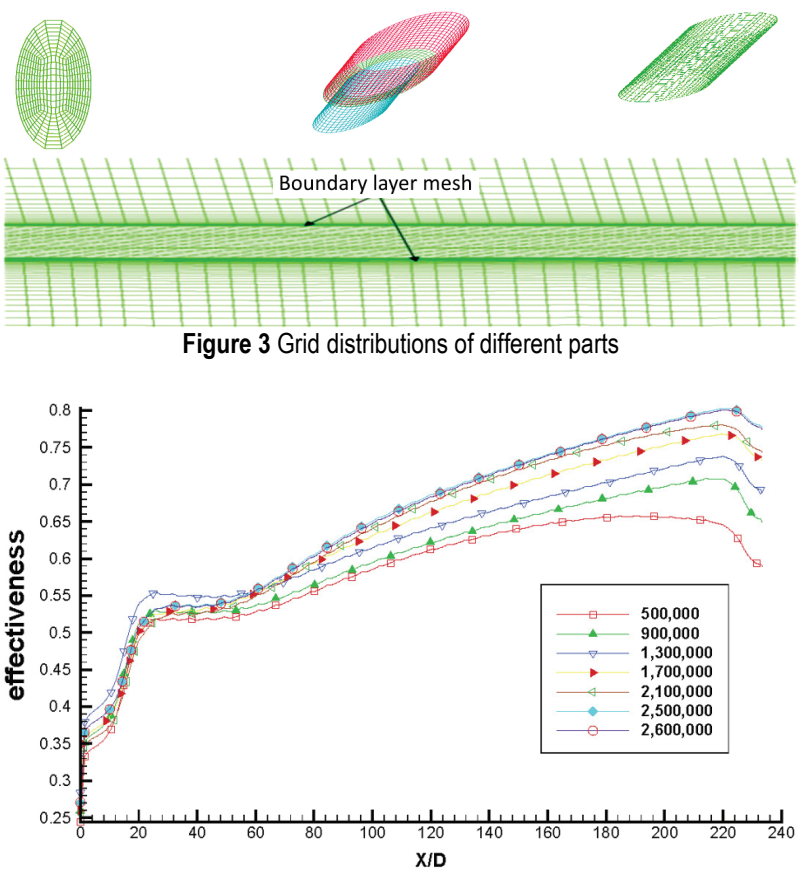

Figure 4 Cooling effectiveness sensitivity in dependence of mesh

The analysis was conducted with element 500000, $900000,1300000,1700000,2100000,2500000$, and 2600000 , of the computational domain. When grids increased to 2500000 , the cooling effectiveness varied little as we tried to increase the number of elements as shown in Fig. 4, in which longitudinal coordinates represented cooling effectiveness, while abscises were presented as $X / D, X$ was the axial distance, $D$ was the equivalent diameter $D_{0}$. Hence, in the different models, the similar meshes were adopted.

\section{COMPUTING DOMAIN AND BOUNDARY CONDITION}

Fig. 5(a) showed the schematic structures of computational domain. The domain covers two columns of holes, the front and back sides were set to periodical boundary, the up side was set as wall, the down side was set as symmetrical, and the coolant and main flow inlets and outlets were set as pressure boundary. Fig. 5(b) shows the detail of hole configuration. The impact platform is marked out and hole distance $P, S$ and incline angle are 
marked on the sketch separately.

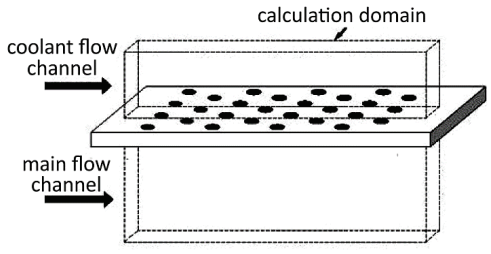

(a)

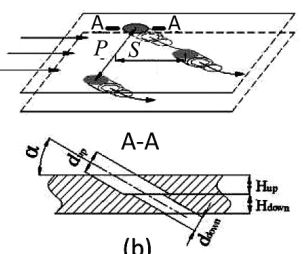

(b)
Figure 5 Sketch of computational domain

ANSYS FLUENT was set to run with double precision and density based solver is used. The working fluid for hot gas and coolant stream was air and assumed as ideal gas. The k-e realizable turbulence model with enhanced wall function was used for solving turbulent quantities because it had better coverage of the convective heat transfer performance predictions [18]. Turbulence intensity of $3 \%$ and $3.1 \%$ was used for coolant and mainstream respectively. The static pressure was set at $1 \mathrm{~atm}$, and the reference to operating pressure of $0 \mathrm{~Pa}$. The mainstream condition was fixed at the entrance with a total pressure of $P_{\text {in }}=32 \mathrm{MPa}$, temperature $T_{\text {in }}=1950 \mathrm{~K}$, coolant flow was fixed at the temperature $T_{\mathrm{c}}=860 \mathrm{~K}$ ). The velocity of the coolant flow $U_{\mathrm{c}}$, is calculated from respective BR (Blowing Ratio) using Eq. (4), which equaled 1.5.

$B R=\frac{\rho_{c} u_{c}}{\rho_{\infty} u_{\infty}}$

where $\rho_{\mathrm{c}}$ and $\rho_{\infty}$ represent densities of the main free stream and coolant, respectively. In addition, $u_{\mathrm{c}}$ and $u_{\infty}$ represented velocities of the main free stream and coolant, respectively.

Mathematically, film cooling effectiveness $(\eta)$ was defined as:

$\eta=\frac{\left(T_{\infty}-T_{\mathrm{w}}\right)}{\left(T_{\infty}-T_{\mathrm{c}}\right)}$

where $T_{\infty}$ represented the main free stream temperature, $T_{\mathrm{w}}$ represented the wall temperature and $T_{\mathrm{c}}$ represented the coolant temperature from hole.

The entrance of the mainstream and coolant was set to pressure inlet while mainstream outlet was set to pressure outlet. All the solid walls have been assumed as no-slip condition. Both sides of the boundary face were set to periodical condition.

\section{NUMERICAL ANALYSIS}

6.1 Comparison between Cylindrical Incline and Stage Incline Holes

\subsubsection{Contribution of Temperature}

Fig. 6 reports the overall temperature maps for the two cooling structures conditions, listed in Tab.1 as Model 1 and Model 2, and in Model 1 Line I going between the two adjacent columns and Line II going right through the hole center were marked separately. The temperature decreased along axial direction, reported the increase of effectiveness [1]. In the upstream region of Model 1, the temperature showed a relative high value, resulting from a poorer previously mentioned effect due to an insufficient coverage of coolant film. However, in the downstream region, the temperature showed a downward trend and irregular distribution. Model 2 had a similar distribution as Model 1, and showed an extension of low temperature region, which was caused by the increasing effect of heat conduction in the steel-made effusion plate.

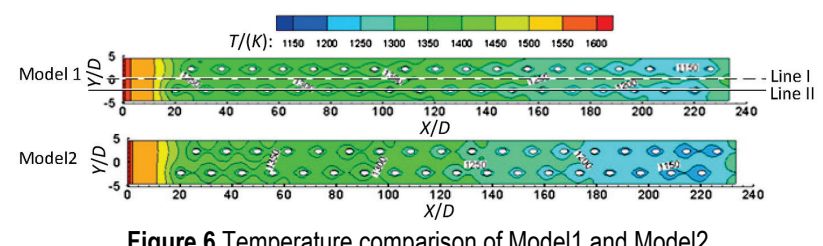

\subsubsection{Contribution of Cooling Effectiveness on Hot Wall}

Fig. 7 shows the distribution of comprehensive cooling effectiveness of Model 1 and Model 2 in Line I and Line II (In Model 1, marked as M1-I, and M1-II, and M2-I, and M2-II in Model 2). The cooling effectiveness increased monotonically and presented a rapid increasing trend in upstream regions, which slowed down in downstream. A faster increasing trending appeared in rear part, which led to superior cooling effectiveness after $X / D=65$, and the results of compound effect had significant film lateral spreading and enhancement convective heat transfer inside the plate.

Fig. 7 shows the cooling effectiveness of Line I on the wall among holes (line M1-II, and M2-II). It decreased first for the film decaying, then increased due to the convective heat transfer of the downstream holes. Model 1 had higher effectiveness in the front part, while Model 2 gradually presents an advantage in the rear.

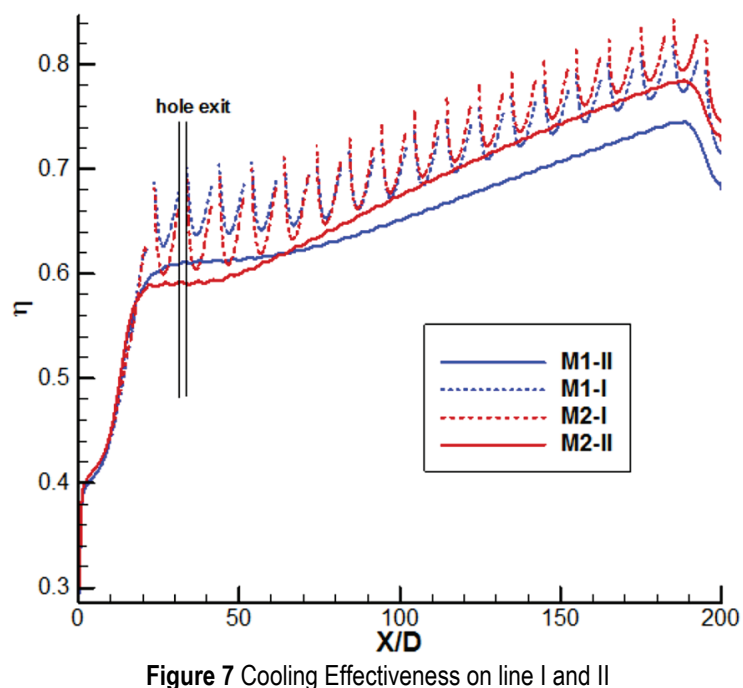

Fig. 8 shows the comprehensive cooling effectiveness on the hole exits on line I. The effectiveness of Modell and Model 2 showed the same trends, with great fluctuation, which was repeated on each film hole exit. The effectiveness of Model 2 was a little lower than of Model 1 , which resulted in the higher temperature on the coolant in Model 2. The stage-hole cooling structure tapped the cooling potential of the coolant to some extent by increasing coolant temperature, obtained superior heat 
transfer between coolant and impact platforms on stagehole walls.

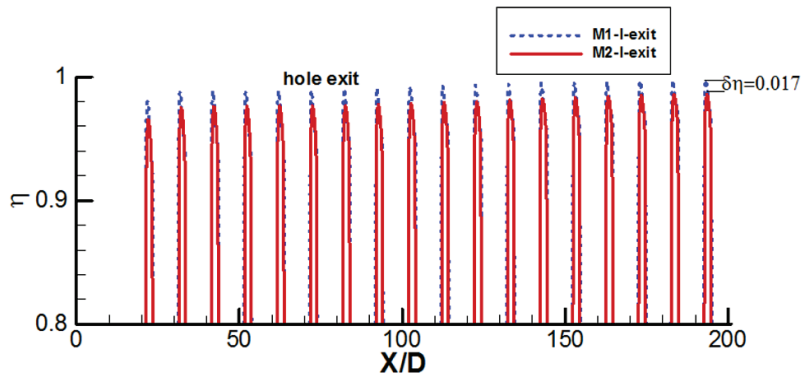

Figure 8 Cooling effectiveness in part of Line II (on the hole exits)

\subsubsection{Contribution of Flow and Mixing}

The cross sections $X=2 D, 4 D, 8 D$ and $16 D$ downstream the hole A, were marked as I, II, III, IV separately, which were shown in Fig.9.

Fig.10(a) and 10(b) showed the maps of temperature in different cross sections $(2 D, 4 D, 8 D$ and $16 D)$, and streamlines (colored by temperature) from hole $\mathrm{A}$ in Model1 and in Model2, the coolant jets flew towards downstream, lifted gradually, and the temperature increased, too.

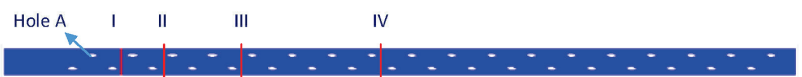

Figure 9 Different sections of downstream

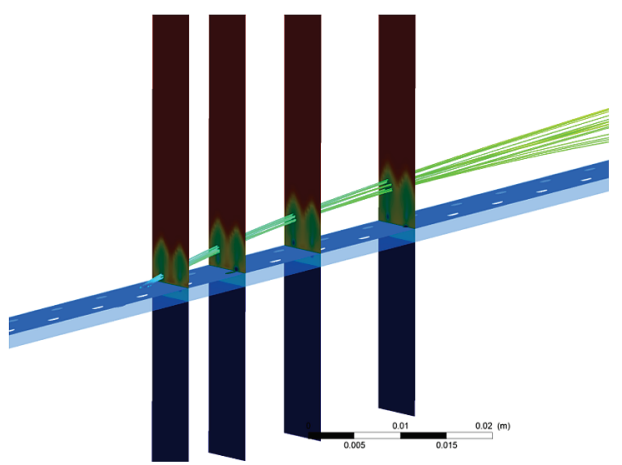

(a) model1

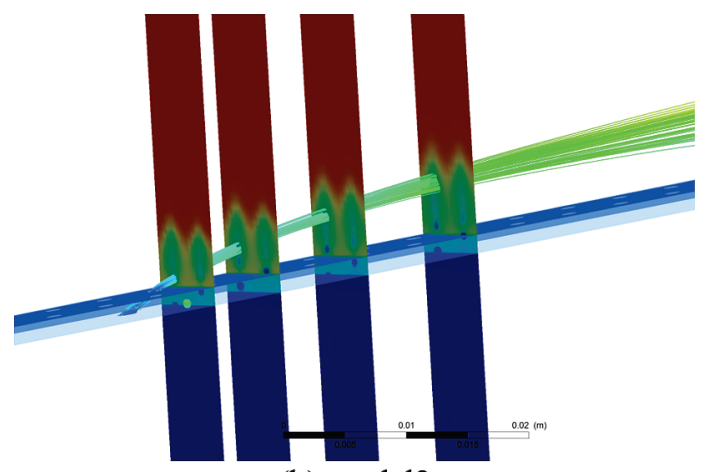

(b) model2

Figure 10 Temperature distributions of downstream cross sections

\section{(A) Flow development downstream the hole}

The distribution of velocity vectors, and local distributions of the temperature (section I) for Model 1 are shown in Fig. 11. The lift off effect caused by the momentum of the coolant led to the kidney vortexes, and the hot gas was induced to flow into the gap between the coolant and the cooling surface by the pressure gradient. Therefore, the lateral spreading of the coolant becomes limiting, which degraded the film-cooling effectiveness, and resulted in a gap between the two vortexes, as shown in circle.

Fig. 11 presents the temperature contour maps and $X$-, $Z$ - components velocity on section I in Model 1 and Model 2 . Both models showed similar distribution, while Model 2 showed wider lateral spreading of the coolant, caused a reduction of high temperature region between two vortexes.

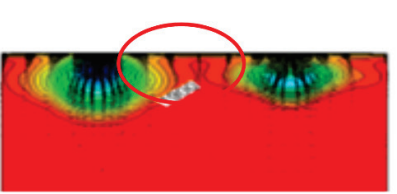

(a) Model2

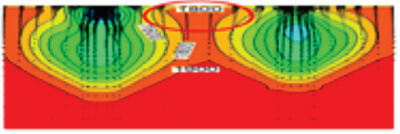

(a) Model2

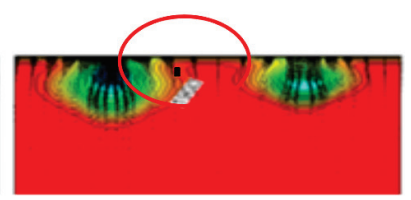

(b) Model1
Figure 12 Kidney and ant kidney vortices in Section

(b) Model1
tices in Section II

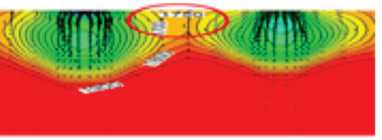

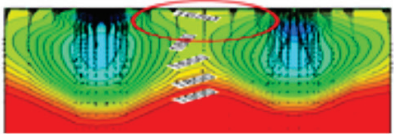

(a) Model2

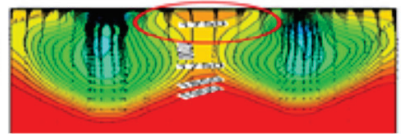

(b) Model1
Figure 13 Kidney and ant kidney vortices in section III

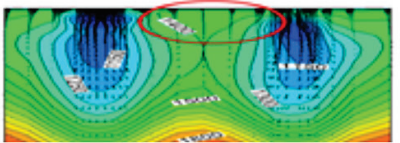

(a) Model2

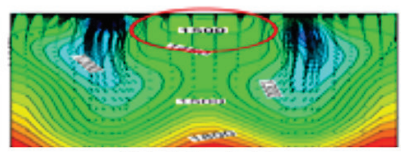

(b) Model1
Figure 14 Kidney and ant kidney vortices in section IV

Figs. 12,13 and 14 present the temperature contour maps and $X$-component, $Z$-component velocity on section II, III and IV in Model 1 and Model 2 separately.

In downstream sections, the staged hole model showed up the advantage gradually. In Fig. 13 and Fig. 14, the kidney vortexes were still there, while the stronger mixing and spreading caused by staged holes in Model 2 resulted in a lower temperature among the vortexes.

Fig. 15 presented the cooling effectiveness distribution of the intersection lines of hot wall and sections I, II, II, IV of Model 1 and Model 2. There appeared two effectiveness peaks in section I (M1-I, M2-II), right downstream the holes both in Model1 and Model 2, and M1-I showed a higher effectiveness. With the increase of distance, in different sections downstream, the regularities distribution was similar, while values increased gradually. It is to be mentioned that the difference between the two models gradually showed a reversal. The effectiveness of Model 2 grew higher than that of Model 1 in section III, and became even larger in downstream section IV, which was consistent of that shown in Fig. 7. 


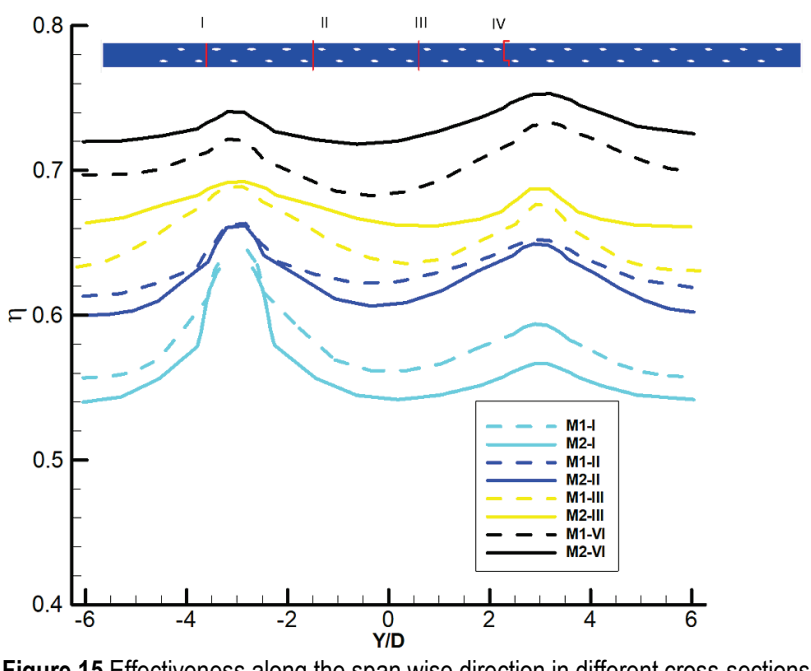

Figure 15 Effectiveness along the span wise direction in different cross-sections

\section{(B) Flow inside holes}

Fig. 16 shows five cross sections inside the hole, marked as I', II', III', IV', and presenting temperature maps and streamlines on different sections.

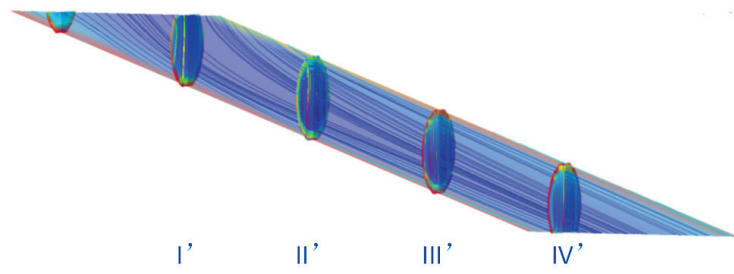

(a) Model 1

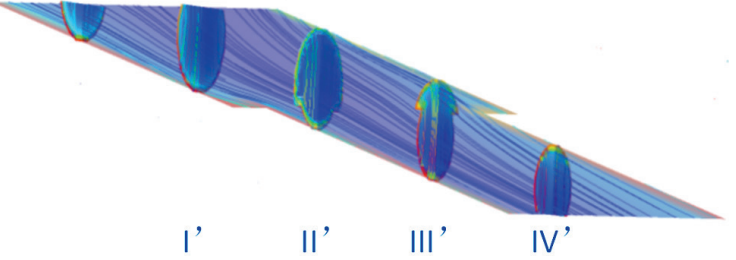

(b) Model 2

Figure 16 Temperatures and streamlines on different sections inside hole

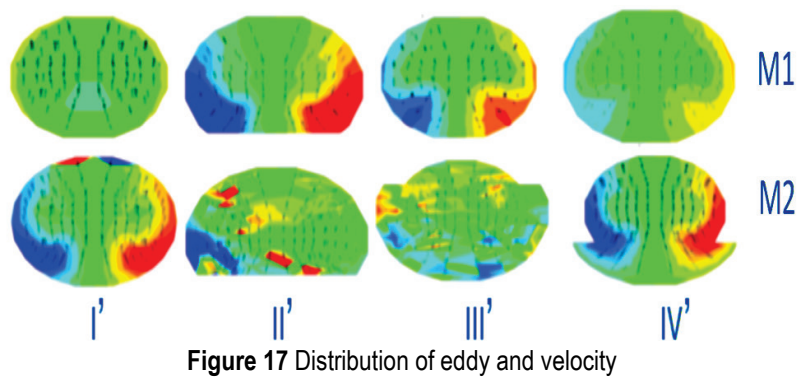

Fig. 17 shows the distribution of eddy, $Y$ - component, and $Z$-component velocity in different sections inside hole. In Model 1, the vortices on section I' are small, and the value is larger in the vicinity of the hole wall, the vortices grow larger, then decrease gradually. In the right side, fluid particles rotate counter clockwise while in the left side they rotate in clockwise direction. In Model 2, compared with Model 1, fluid particles show stronger rotation speed in section I', while the distribution did not show any regular pattern in section II' and III', which was caused by the impingement on the platform. In section IV', the distribution was restored to be regular, and the vortices were even larger, especially in the vicinity of the hole wall, meaning stronger rotating speed, which induced the stronger span spreading downstream.

Fig. 18 shows the distribution of turbulent kinetic energy in different sections. It could be seen that the fluid obtained larger turbulent kinetic energy near the hole wall in section I', caused by the larger impingement speed. In addition, the energy gradually dissipated towards the center, resulting in a lower value region, which meant the fluid restored to be regular gradually. In section II', the turbulent kinetic energy became larger gradually in the lower region, and indicated a certain gradient, while it became smaller in the upper region. With distance increased, the turbulent kinetic energy distribution tended to be uniform, and the gradient decreased gradually. In section IV', the distribution was like that of section I', and the value increased first and then decreased gradually in the hole, meaning that the flow was disordered and then gradually stabilized.
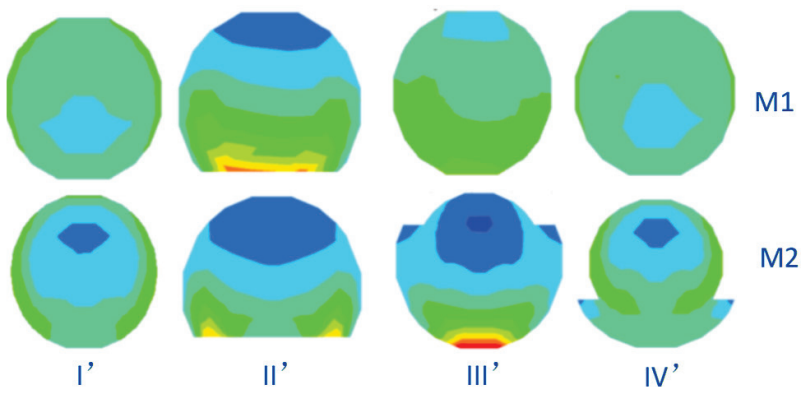

Figure 18 Distribution of turbulent kidney energy

In Fig. 18, the turbulent kinetic energy of Model 2 showed a different distribution from Model 1. In section I', the low turbulent kinetic energy region of Model 2 was on the upper region. Further, the high turbulent kinetic energy region was divided into two regions in section II' in Model 2, which was caused by the effect of the platform barrier. Finally, in section IV', the Model 2 obtained higher value on both sides near the hole wall than Model1, meaning strong flow speed near both sides.

\section{DISTRIBUTION OF VELOCITY IN HOLE EXIT}

Fig. 19 shows the pressure and $X$-component and $Y$ component velocity distribution in hole exit planes. In Model 1, the velocity formed a clockwise vortex on the left and a clock vortex on the right above center axis, marked as I and II, and under center axis there were also two vortexes, marked as III and IV. Both the vortexes under center axis rotated in the opposite direction of the vortexes above. However, Model 2 also induced four similar vortexes (I', II', III', IV') in corresponding places, and resulted in a bigger effecting area and higher intensity of the vortexes.

The complicated flow characteristics inside hole led to complex flow distribution of the exit, enhanced the vortex intensity, and strengthened the film span wise spreading.

To get a view as complete as possible of the specific effects of varying hole shapes inside and hence an enhanced understanding of the dominating mechanisms, a variation of the hole geometry was imperative, and the area ratios and height ratios of holes were considered to be the 
most important geometric parameters. The effect of changing of exactly these parameters on the film cooling performance will be analyzed.

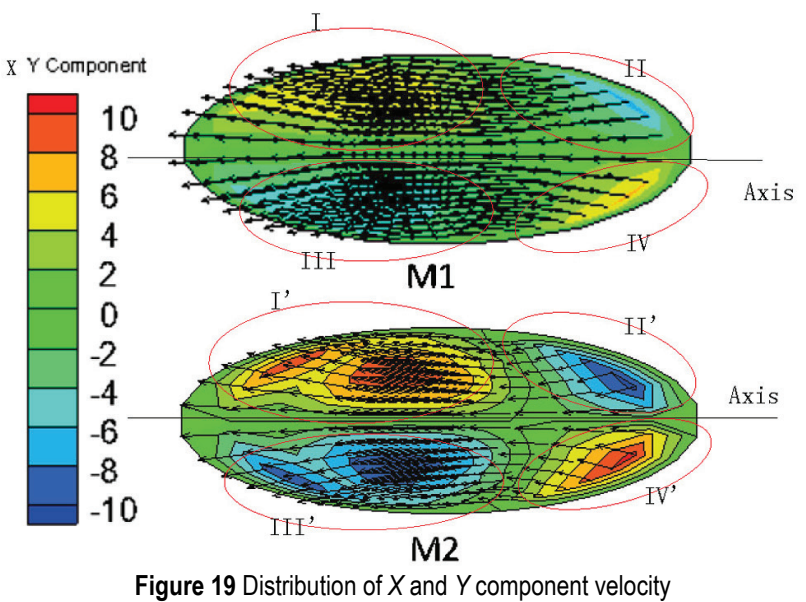

\subsection{Influence of the Different Area Ratio}

The investigations on the effect of different area ratios have been conducted. Fig. 20 shows the temperature contour maps of the Model 2, 3, 4, with increasing area ratios (Tab. 1). Those had similar temperature contour maps, especially in the front of the plates, while in the rear of the plates, the temperature decreased in turn because of the increasing of convective heat transfer inside the plate.

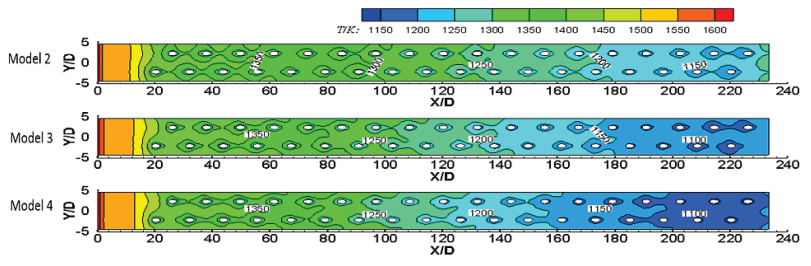

Figure 20 Distribution of temperature contours

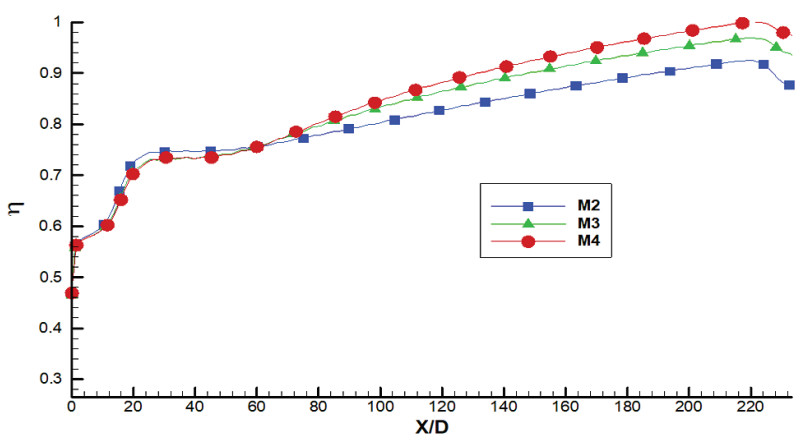

Figure 21 Distribution of cooling effectiveness on Line I

Fig. 21 shows the distribution of comprehensive cooling effectiveness on line I (seen in Fig. 6). The models show similar increasing trends as Model 2, and the value increased with the area ratios, while the difference enlarged towards downstream.

Fig. 22 shows the temperature contour maps and Xand Z-components velocity in section I, II, and III. The spreading ability of Model 2, 3, 4 decreased in turn, and the difference among the three models increased gradually in cross section I, section II, and section III. The flow kinetic energy dissipated in impinging process on the stage flats, which grew even larger inside models with large area ratio.
The impinging cooling ability grown larger resulted in high cooling effectiveness and strengthened unsterilized flow. Those led to wonderful lateral spreading, and minimized the gap between two vortexes.
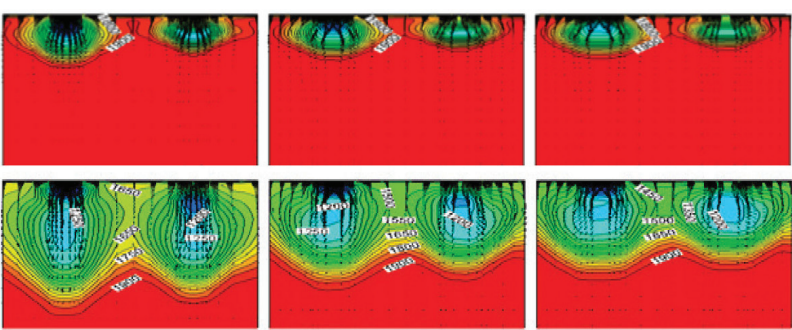

Model2 Model3

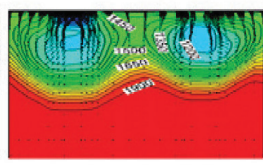

Figure 22 Distribution of temperature and velocity vectors

\subsection{Influence of the Different Height Ratio}

The investigations on influence of different height ratios have been conducted. Fig. 23 shows the temperature contour maps of the Model 2, 5, 6, with increasing height ratios (Tab. 1). The three models had nearly the same temperature maps those proved height ratio had little affect on heat transfer.

Fig. 24 shows the distribution of comprehensive cooling effectiveness on Line I, and the models show almost the same trends as Model 2. It could be further concluded that height ratio effected slightly.

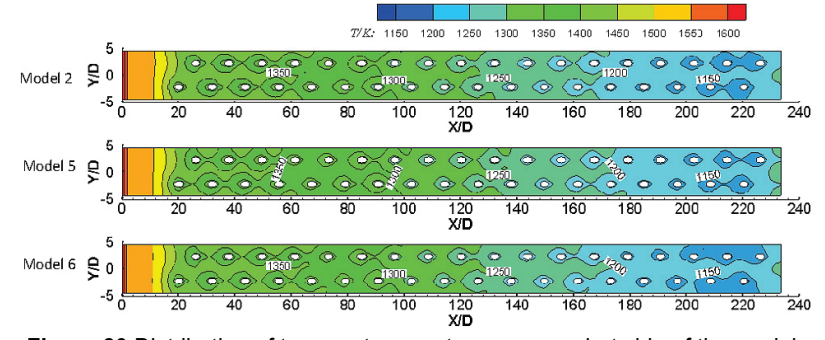

Figure 23 Distribution of temperature contour maps on hot side of the models

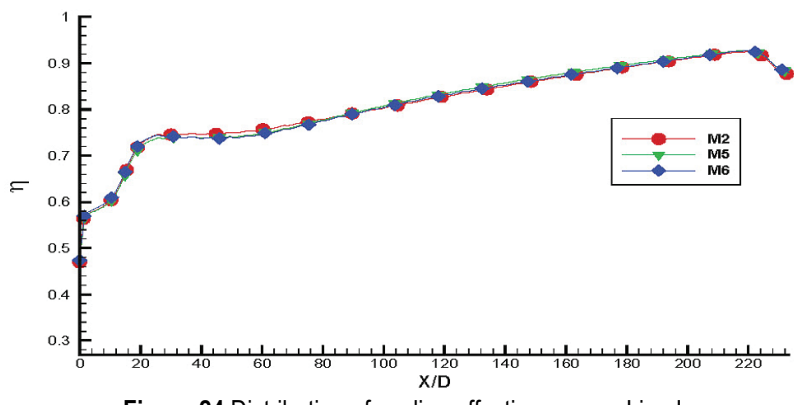

Figure 24 Distribution of cooling effectiveness on Line I

\section{CONCLUSIONS}

In this paper, the flow and heat transfer characteristics of the stage orifice plate have been numerically studied and compared with the equivalent flow area cylindrical orifice plate at the same flow ratio. In addition, the effects of area ratio and height ratio have been studied.

(1) The impact platform induced into the effusion wall gave a raise of the intensity of secondary flow, resulting in an increase in the complexity of its distribution. Those resulted in superior heat transfer in holes, and strengthened lateral spreading in downstream of the hole exits. 
(2) Stage hole effusion plates induced an increasing of cooling effectiveness, especially in the rear part. Further, that led to an improvement of lateral uniformity of temperature, which proved to be caused by superior impingement inside the wall and stronger film lateral spreading on the surface.

(3) Inducing the stage holes, stage hole showed a convective heat transfer enhancement between the solid wall and coolant gas, then induced an increasing of coolant temperature, and made a tap of the cooling potential of the coolant to some extent.

(4) The area ratio has a great influence on the flow and heat transfer characteristics, and the high area ratio always causes efficient cooling, while the height has a slight influence on the cooling effect.

\section{REFERENCES}

[1] Goldstein, R. J., Eckert, E. R. G., \& Burggraf, F. (1974) Effects of hole geometry and density on three-dimensional film cooling. International Journal of Heat and Mass Transfer, 17(5), 595-607. https://doi.org/10.1016/0017-9310(74)90007-6

[2] Becchi, R., Facchini, B., Picchi, A., et al. (2015). Film cooling adiabatic effectiveness measurements of pressure side trailing edge cooling configurations. Propulsion \& Power Research, 4(4), 190-201. https://doi.org/10.1016/j.jppr.2015.10.001

[3] Cerri, G., Giovannelli, A., Battisti, L., et al. (2007). Advances in effusive cooling techniques of gas turbines. Applied Thermal Engineering, 27(4), 692-698. https://doi.org/10.1016/j.applthermaleng.2006.10.012

[4] Kim, S. M., Lee, K. D., Kim, K. Y. (2011). Numerical Study on Film-Cooling Effectiveness for Various Film-Cooling Hole Schemes. Journal of Computational Fluids Engineering, 16(4), 92-99. https://doi.org/10.6112/kscfe.2011.16.4.092

[5] Qu, L., Zhang, J., \& Tan, X. (2017). Improvement on film cooling effectiveness by a combined slot-effusion scheme. Applied Thermal Engineering, 126, 379-392. https://doi.org/10.1016/j.applthermaleng.2017.07.188

[6] Andreini, A., Facchini, B., Becchi, R., et al. (2016). Effect of slot injection and effusion array on the liner heat transfer coefficient of a scaled lean-burn combustor with representative swirling flow. Journal of Engineering for Gas Turbines and Power, 138(4), 041501. https://doi.org/10.1115/1.4031434

[7] Sidik, C., Azwadi, N., \& Ehsan, K. (2014). Computational Investigation of Film Cooling from Cylindrical and Row Trenched Cooling Holes near the Combustor End Wall. Applied Mechanics and Materials. Trans Tech Publications, 554, 225-229.

[8] Ligrani, P., Goodro, M., Fox, M., et al. (2012). Full-coverage film cooling: film effectiveness and heat transfer coefficients for dense and sparse hole arrays at different blowing ratios. Journal of Turbomachinery, 134(6), 061039. https://doi.org/10.1115/1.4006304

[9] Schulz, A. (2001). Combustor liner cooling technology in scope of reduced pollutant formation and rising thermal efficiencies. Annals of the New York Academy of Sciences, 934(1), 135-146. https://doi.org/10.1111/j.1749-6632.2001.tb05848.x

[10] Kelly, G. B. \& Bogard, D. G. (2003). An investigation of the heat transfer for full coverage film cooling. ASME Turbo Expo 2003, collocated with the 2003 International Joint Power Generation Conference. American Society of Mechanical Engineers, 581-590. https://doi.org/10.1115/GT2003-38716
[11] Krewinkel, R. (2013). A review of gas turbine effusion cooling studies. International Journal of Heat and Mass Transfer, 66, 706-722. https://doi.org/10.1016/j.jiheatmasstransfer.2013.07.071

[12] Andrews, G. E., Asere, A. A., Gupta M. L., et al. (1990). Effusion cooling: the influence of the number of holes. Proceedings of the Institution of Mechanical Engineers, Part A: Journal of Power and Energy, 204(3), 175-182. https://doi.org/10.1243/PIME_PROC_1990_204_024_02

[13] Murray, A., Ireland, P., Wong, T., et al. (2018). High Resolution Experimental and Computational Methods for Modelling Multiple Row Effusion Cooling Performance. International Journal of Turbomachinery, Propulsion and Power, 3(1), 4. https://doi.org/10.3390/ijtpp3010004

[14] Cho, H. H. \& Rhee, D. H. (2001). Local heat/mass transfer measurement on the effusion plate in impingement/effusion cooling systems. Journal of Turbomachinery, 123(3), 601608. https://doi.org/10.1115/1.1344904

[15] Huber, A. M. \& Viskanta, R. (1994). Effect of jet-jet spacing on convective heat transfer to confined, impinging arrays of axisymmetric air jets. International Journal of Heat and Mass Transfer, 37(18), 2859-2869. https://doi.org/10.1016/0017-9310(94)90340-9

[16] Rhee, D. H., Choi, J. H., Cho, H. H. (2003). Flow and heat (mass) transfer characteristics in an impingement/effusion cooling system with crossflow. Transactions of the ASMET-Journal of Turbomachinery, 125(1), 74-82. https://doi.org/10.1115/1.1519835

[17] Kim, K. M., Moon, H., Park, J. S., et al. (2014). Optimal design of impinging jets in an impingement/effusion cooling system. Energy, 66, 839-848. https://doi.org/10.1016/j.energy.2013.12.024

[18] ANSYS Fluent Tutorial Guide, ANSYS, Inc. Release 17.0 Southpointe, http://www.ansys.com

\section{Contact information:}

Bo ZHANG, Corresponding author

Jiangsu Province Key Laboratory of Aerospace Power System,

College of Energy and Power, Nanjing University of Aeronautics and

Astronautics, Nanjing 210016, China

Email: zhangbo_nuaa@163.com

\section{Yuanyuan DOU}

Jiangsu Province Key Laboratory of Aerospace Power System,

College of Energy and Power, Nanjing University of Aeronautics and

Astronautics, Nanjing 210016, China

\section{Quan HONG}

Jiangsu Province Key Laboratory of Aerospace Power System,

College of Energy and Power, Nanjing University of Aeronautics and Astronautics, Nanjing 210016, China

\section{Honghu Jl}

Jiangsu Province Key Laboratory of Aerospace Power System,

College of Energy and Power, Nanjing University of Aeronautics and Astronautics, Nanjing 210016, China 